International Journal of Dental Research, $8(2)(2021) 42-48$
International Journal of Dental Research
SPC
Website: www.sciencepubco.com/index.php/IJDR
Research paper

\title{
Oral hygiene and dietary choices of 8-11year-old children with respect to the evidence-based oral health messages (diet and tooth brushing) in 3rd and 4th class children in primary schools in cork, Ireland, and their relationship to BMI taking account of their physical activity. "Cross- sectional study"
}

\author{
Ahmed Mohamed ${ }^{1 *}$, Dr. Mairead Harding ${ }^{1}$, Dr. Janas Harrington ${ }^{1}$ \\ ${ }^{1}$ Department of preventive and community dentistry, Sebha University, Sebha, Libya \\ *Corresponding author E-mail: ahmedglewan@gmail.com
}

\begin{abstract}
Background: Dental caries and obesity are growing public health conditions over the word. Objective: The aim of this study was to determine the prevalence of being overweight and obese amongst 8-11-year-old children in Cork city and county, and whether there is an association with oral hygiene practices, dietary choices, and BMI.

Methods: This is a cross-sectional analysis using secondary data. Data came from the Cork Children's Lifestyle Study (CCLaS); were analysed using logistic regression and controlling for potential confounder for 1061 children aged 8-11 years.

Result: This study included 1,061 children, 58\% of the sample were boys at median age 9 years (8-11 years). There was an association between BMI and toothbrushing $(\mathrm{P}>0.005)$. The relationship between BMI and diet frequency (breakfast, dinner meals and favorite snack) was statistically significant. Regarding diet quality, Junk food was only found statistically significant associated with BMI. While the relationship between BMI and diet quantity, several variables were found statistically significant associated with BMI include (Sum) Kcalall-foods, total sugar from all include all beverages per day, and fibreaoac.

Conclusion: The data from CCLaS suggested that the negative association between BMI and toothbrushing frequency in children aged 8 to 11 years-old requires further study. Dietary choices, especially sweets have an important role in being overweight and suffering obesity as indicated in this study and sugars are the principle etiological cause of dental caries. Therefore, strategies should be developed on a multidisciplinary level with a common risk factor approach.
\end{abstract}

Keywords: BMI 'Body Mass Index'; Dental Caries 'Tooth Decay; Diet; School Children.

\section{Introduction}

Dental caries and obesity are growing public health conditions (Reilly et al., 2009). Dental caries is still a major public health problem in high-income countries and the burden of dental caries is growing in middle- and low-income countries. The worldwide incidence of dental caries has increased and subsequently leads to a negative effect on individuals and communities in terms of pain, suffering, impairment of function, and reduced quality of life (Pitts et al., 2011). Dental caries (tooth decay) is a multi-factorial disease that leads to the breakdown of tooth structure and if left untreated leads to pain and sepsis. Caries is created by a complex process over time between cariogenic bacteria, fermentable carbohydrate, tooth surfaces and other host factors, such as genetic, behavioral, and environmental factors.

\subsection{Bacteria: The bacteria is a main component of caries process}

Which accumulates and colonizes on tooth surface in a biofilm (dental plaque). It produces acid from carbohydrate, which leads to demineralization of the tooth substance and induction of a cavity on the surface (Selwitz et al., 2007). Cariogenic bacteria includes mutants streptococci, Streptococcus sobrinus and Lactobacillus spp exist in dental biofilm. Mutant Streptococci (M. Streptococci) is the main cariogenic bacteria in the oral cavity and is highly concentrated in biofilm (dental plaque). It produces weak organic acids as a result of fermentable carbohydrates metabolism. This acid leads to drop local $\mathrm{pH}$ values below a critical level and results in demineralisation of tooth tissues. If calcium, phosphates, and carbonate diffusion is continued without intervention, the caries lesion (tooth cavity) occurs. Caries occurs in pits and fissures, as well as on smooth surfaces of the tooth (Selwitz et al., 2007). The presence and the extent of M. 
Streptococci in children are indicators of caries risk. Other microorganisms, such streptococcus salivarius, S. sanguis and lactobacillus casei, also contributed to the dental caries process and usually exist at high concentration in dentine and root caries (Council, 1989).

\subsection{Diet: Diet is another principle component of the dental caries process}

Although it is undeniable that sugar has clearly been correlated to dental caries for several years, modern diets, which typically contain a wide range of fermented carbohydrates (sugar), play an important role in increasing dental caries (Gupta et al., 2013). In addition, the characteristics of sugars like starch and fiber forms have a significant impact in creating obesity, because of their complexity and storage in the body in fat forms. From the dental perspective, these forms of sugar have a clear effect on developing dental caries due to their consistency and adherence to the tooth surface and their ability to drop mouth pH to the critical level (Henriksen and Kolset, 2007). Such elements of modern diets include highly processed starch-containing foods and foods that contain novel synthetic carbohydrates (Gupta et al., 2013). Fermentable carbohydrates (sugars) includes monosaccharide (glucose, fructose, and galactose) and disaccharide (sucrose, lactose, and maltose). Sucrose is high potential cariogenic, and has ability to support synthesis of extracellular glucans in dental biofilm by bacteria (M. Streptococci), and promotes its accumulation on tooth surface. This sort of sugar is easily metabolised by bacteria and produces acid that leads to reduction of oral $\mathrm{pH}$ to critical value and demineralisation of tooth tissue (Gupta et al., 2013; Jeor et al., 2001).

\section{Methods}

Data collected in the Cork Children's Lifestyle Study (CCLaS); a cross-sectional survey of 3rd and 4th class children (8-11 year old children) in primary schools in Cork, Ireland will be used in this research. This research project will assess the toothbrushing habits and dietary choices of 8-11 year old children with respect to the evidence-based oral health messages.

These include: (i) reduce the consumption frequency and amount of sugar containing foods, (ii) reduce the frequency and amount of acidic drinks, (iii) promote tooth brushing, and investigate whether a relationship to BMI exists (taking into account the participant's level of physical activity).

\subsection{Study population}

Data were collected from the Cork Children Lifestyle Study (CCLaS). The sample involved participants from schools (51 schools), including all primary schools in Cork and Mitchelstown, while excluding special needs schools and schools without the target class/age. The main target population was 3rd and 4th classes at age 8-11 years. A total number of 1,060 children whose parents/guardians provided consent and who completed the questionnaires were included. BMI is defined as a dependent variable, and defined in the following categories: underweight (<18), normal weight (18-24.9), overweight (25-29.5), and obese (>30).

Two trained researchers measured the BMI recordings (weight divided by height squared). A Leicester portable height stick was used to measure the height of the participants without shoes to the nearest $\mathrm{mm}$. The study children's weights were determined without shoes and in light clothing, using Tanita WB100MA mechanic scales and measured to the nearest $0.1 \mathrm{~kg}$.

\subsubsection{Data collection}

Data collection took place between April 2012 and June 2013. Firstly, permission was obtained from the principals (school heads), and then the participants' consents were obtained by handing the consent forms along with an information letter to children to take home to their parents/guardians. After consent was granted, data were collected via a self-administrated questionnaire distributed to the parents/guardians, and children completed a questionnaire with the assistance of the researchers.

\subsubsection{Dietary choices}

24 choices covered three days and the breakfast each morning, which was explained by using a poster template of the food diary.

\subsubsection{Questionnaire data}

The questionnaire was developed, tested, and all questions were validated, including individual, family and environmental factors. The questionnaire was divided into the following sections: Principal's questionnaire The School Principal in each school was asked to complete the questionnaire containing questions under six main headings: (1) Demographics. (2) Health curriculum. (3) School policy environment. (4) Level of nutritional care. (5) Provision of physical activity. (6) Parental/community support.

\subsubsection{Children's questionnaire}

The child questionnaire contained information under five main headings: (1) Background information. (2) Your neighborhood. (3) Food and diet. (4) Sports and physical activity. (5) Hobbies and activities. The parent /guardian's questionnaire included questions categorised into nine major headings: (1) study child's birth factors. (2) Study child's current health. (3) Study child's exercise and physical activity. (4) Study child's hobbies and activities. (5) Study child's diet and dietary habits. (6) Current parental health. (7) Parental diet. (8) General family eating questions. (9) Family background.

\subsubsection{Dietary intake}

The food diary was described to children in the classroom using a poster template and they were taught how to complete each meal section and how to use the food atlas photographs to estimate the portion size. The dietary assessment included three consecutive days and each day was broken down into six meal sections: (1) Breakfast. (2) Morning snack. (3) Lunch. (4) Afternoon snack. (5) Dinner. (6) Evening snack.

The six primary questions that were asked in each meal section include: (1) Time meal/snack was consumed. (2) Location where the meal was consumed. (3) Type of food or drink consumed. (4) Quantity of food or drink consumed. (5) Quantity leftover. (6) Cooking method used. 


\subsubsection{Physical activity}

Physical activity was calculated using a GENE active accelerometer (a small, lightweight, waterproof device) for seven consecutive days . The accelerometer manufacturer adjusted the unit before the study commenced to record the data at $100 \mathrm{~Hz}$ for seven days. The children were instructed to wear the accelerometer the whole day (day and night) and took it off just if their coach asked them to do so during sports activities. The accelerometer was placed on the wrist of the non-dominant hand, and the research assistants recorded information of which hand they normally used. The data were transformed into one second and one minute formats for data analysis and downloaded in "csv" and "bin" formats. The output measures were; amount of sedentary time and the amount of low, moderate, and vigorous activity per day.

\subsection{Statistical analysis}

Statistical analysis was performed using Statistical Package of Social Science (SPSS version 20). Descriptive analyses were used to describe: (1) the prevalence of overweight and obese participants. (2) The frequency and the amount of foods consumed. (3) Tooth brushing frequency. Frequency and percentages are reported for categorical data. For continuous data, the mean and standard deviation were calculated when data were normally distributed, while the median with interquartile (Q1 and Q3) values are reported for non-normally distributed data. The association between BMI and dietary choice, tooth-brushing frequency were examined using linear regression analysis for continuous data and categorical regression for categorical data (nominal data). For whole prediction, R square and ANOVA is calculated with the mean difference between each category $(\mathrm{df})$ for categorical data, while coefficient correlation and $\mathrm{P}$ value $(\beta$ and $\mathrm{P}<0.05)$ are reported for each specific variable. Bivariate correlation is calculated and Pearson $r$ is reported (between -1 and +1 ). Then the significant variables are adjusted for potential covariates (physical activity). Some variables (string data) were only described without assessing their relationship to BMI, these variables such as type of snacks and favorite dinks were used as explanatory data only to show what kind of snacks and drinks children consumed.

\section{Result}

\subsection{Subjects characteristics}

This study included 1,061 children, $58 \%$ of the sample were boys at median age 9 years (8-11 years). The participants' BMI readings were at 17.64 median (16.13-19.87). According to the International Obesity Task Force, which indicates normal weight (18-24.9), overweight (25-29.9) and obese (> 30), about $74.7 \%$ of the children had a normal weight while $20.0 \%$ were overweight and just $5.3 \%$ obese. There was no data on underweight children.

\subsection{Correlation between BMI and oral hygiene}

The oral hygiene status was recorded using the parent's questionnaire; parents were asked how often their children's teeth were brushed. A high proportion of children brush twice a day (64\%), while fewer (28\%) brush once a day, and $2 \%$ brush more than twice a day. Furthermore, $5 \%$ reported that they brushed less than once a day and $<1 \%$ do not brush.

Bivariate correlation was calculated for BMI and reported tooth-brushing frequency. The data revealed a negative correlation between BMI and oral hygiene (tooth brushing) is contributing in prediction of BMI by $18 \%$ (Pearson $r=-0.018$ ) and it is statistically none significant $(\mathrm{P}=0.577)$ (Table 1). Thus, there is a negative association between BMI and tooth brushing status, but it is not statistically significant.

Table 1: correlation between BMI and Tooth brushing

\begin{tabular}{lll}
\hline Variable & correlation & Significance \\
\hline Tooth brushing frequency & -0.018 & 0.577 \\
\hline$(*)$ for significant variables $(\mathrm{P}$ value $<0.05)$ & &
\end{tabular}

\subsection{Correlation between BMI and diet frequency}

Bivariate correlation was performed to assess the relationship between BMI and diet frequency. The parents' response was calculated separately from the children response. The data revealed that children eating breakfast at mean 18.19 (SD2.9), who having meal at evening at mean 18.24 (SD 2.939). Children whom sit on table for evening meal at mean 18.25 (SD2.976). The data showed also children who eat breakfast every day at mean 18.04 (SD2.813) while who never eat breakfast at 34 mean 20.12 (SD 2.796). Participants those had snacks between meals at mean 18.35 (SD 3.054) and those had snacks every day at mean 17.76 (SD 2.858). There is a positive relationship between BMI and breakfast meal (Pearson $\mathrm{r}=0.069$ and $\mathrm{p}=0.28$ ), also the relationship between BMI and evening meal showed a positive relationship (Pearson $\mathrm{r}=0.125$ and $\mathrm{p}=0.000$ ). Thus breakfast and evening meals are predicting BMI changes by $6.9 \%$ and $12.5 \%$ respectively) (table 2).

Table2: Model 1 Correlation between BMI and Diet Frequency

\begin{tabular}{lll}
\hline Questions & Correlation & Significant \\
\hline Breakfast (Q1 parents' response) & 0.069 & $.028^{*}$ \\
$\begin{array}{l}\text { Evening meals (Q2 } \\
\text { parent response) }\end{array}$ & 0.125 & $.000^{* * *}$ \\
$\begin{array}{l}\text { Sit at a table for the evening meal (Q3 } \\
\text { parents' response) }\end{array}$ & -0.020 \\
\hline
\end{tabular}

(*) for significant variables $(\mathrm{P}$ value $<0.05)$

Favorite snacks (Q3 children's response), that include biscuits/cakes, scones, chocolates, yoghurts, crisps/popcorn/pretzels, and nuts, which were only explained in descriptive analysis to show sort of snacks were consumed. However, in children response, the relationship between favorite snacks frequency and BMI revealed a significant correlation. This contributes most and this association is statistically significant (Pearson $\mathrm{r}=-0.90, \mathrm{P}$ value=0.04). However, when children were asked about snacks (the dichotomous variable) between meals in the day before (Q2 children response) the results showed a non-significant association (P value=0.935) (Table 3). 
Table3: Model 2 Correlation between BMI and Diet Frequency

\begin{tabular}{lll}
\hline Questions & Correlations & Sign \\
\hline Breakfast frequency (1Q children response) & -0.021 \\
snacks (Q2 children response) & 0.003 \\
Favourite snack (Q3 children response) & 0.90 \\
Favourite drink (Q4 children response) & 0.022 & 0.935 \\
\hline
\end{tabular}

Favourite drink (Q4 children response)

(*) for significant variables $(\mathrm{P}$ value $<0.05)$

The relationship between BMI and favorite drink frequency (Q4 Children response) is not statistically significant ( $p>0.005)$ (Table 3). Hence, the breakfast, evening meal and favourite snack frequency are positively associated with BMI changes.

\subsection{Correlation between BMI and diet quality}

The relationship between BMI and diet quality was evaluated using multiple categorical regression. The overall predication of the food quality model is $1.7 \%$ (Adjusted $\mathrm{R}$ square $=0.017$ ), with mean squared (2.014) and this is not statistically significant (ANOVA $\mathrm{P}$ value $=0.009)($ Table 20). Coefficient correlation showed that fruits and milk types are negatively associated with BMI $(\beta=-.023$ and 0.009), with mean differences between categories (df 3 fruits and 1 milk types), but this is not statistically significant ( $\mathrm{P}$ value $>0.005)$. Unhealthy diets including junk food predicts BMI by about $16 \%$ with mean difference between each category (df 5), and this association is statistically significant $(\mathrm{P}$ value $=0.00)$. High fat food contributes in predication of BMI by over than $30 \%$, but is not statistically significant (P value $>0.05$ ) (Table 4).

Table 4: Coefficient Correlation between BMI and Diet Quality

\begin{tabular}{llll}
\hline Questions & Coefficient correlation Standardised Beta & Df & Significant \\
\hline Fruits & -0.023 & 2 & 0.384 \\
Vegetables & 0.058 & 3 & 0.313 \\
Milk types & -0.009 & 1 & 0.904 \\
Sweets & -0.023 & 3 & 0.934 \\
High fat food & -0.037 & 1 & 0.749 \\
Sweets as reward & 0.019 & 1 & 0.778 \\
Junk food & 0.169 & 5 & $0.000 * * *$ \\
\hline
\end{tabular}

$(*)$ for significant variables $(\mathrm{P}$ value $<0.05)$

\subsection{Correlation between BMI and diet quantity}

The correlation between BMI and diet quantity is evaluated by multiple linear regression. The Model 1 summary for overall predicators is $3.9 \%$ ( $\mathrm{R}$ square $=0.039)$, but it is not statistically significant $(\mathrm{P}$ value $=0.169)$. The coefficient correlation shows sugar fizzy drinks and daily sugar fizzy drinks contribute in predication of BMI $(\beta=--3.359$ and 3.523, respectively). The total sugar including all beverages per day contributes in BMI prediction by $35 \%(\beta=0.353)$ and is statistically significant (P value $=0.012)$. Sum Kcal-all-foods is also statistically significant (P value 0.015$)$ and predicts BMI by $61.0 \%(\beta=.610)$. While sugar fizzy drinks and daily sugar fizzy drinks, are not statistically significant (P value 0.631 and 0.627 ). The total sugar from all food including all beverages per day and Sum Kcal-all-foods, are strongly and positively associated with BMI (Table 5).

Table 5: Model 1 Coefficient Correlations between BMI and Diet Quantity

\begin{tabular}{|c|c|c|c|c|}
\hline \multirow{2}{*}{ Questions } & \multirow{2}{*}{ Coefficient correlation Standardized Beta } & \multirow{2}{*}{ Significant } & \multicolumn{2}{|c|}{ Confident Interval CI (95\%) } \\
\hline & & & Upper & Lower \\
\hline Snacks & .087 & .078 & 0.00 & .004 \\
\hline (Sum) Soft drink bottle per week & .015 & .766 & -.251 & .341 \\
\hline (Sum) Soft drink cans per week & .070 & .149 & -.51 & .332 \\
\hline (Sum) Energy drink bottles per week & .058 & .376 & -.233 & .617 \\
\hline (Sum) Kcalall-foods & 610 & $.015^{* *}$ & .000 & .003 \\
\hline Sugar from all foods incl all beverages per day & .353 & $.012 * *$ & .005 & .041 \\
\hline
\end{tabular}

(*) for significant variables $(\mathrm{P}$ value $<0.05)$

In model 2 of diet quantity, the other components of diet were assessed. The whole model contributes to BMI by 15\% (sign ANOVA .003). The saturated fat predicts the BMI most among the other components, but it is not statistically significant ( $\mathrm{p}$ value $=-.298)$. The fibreaoac was the only statistically significant factor that correlated to BMI ( $\mathrm{p}$ value $=0.020$ ). It is negatively associate with BMI, where BMI increased, the fibreaoac decreased (Table 6).

Table 6: Model 2 Coefficient Correlations between BMI and Diet Quantity

\begin{tabular}{|c|c|c|c|c|}
\hline \multirow{2}{*}{ Questions } & \multirow{2}{*}{ Coefficient correlation Standardized Beta } & \multirow{2}{*}{ Significant } & \multicolumn{2}{|c|}{ Confident Interval CI (95\%) } \\
\hline & & & Upper & Lower \\
\hline (Sum) protein & .060 & .222 & -.002 & .007 \\
\hline (Sum) carbohydrate & -.086 & .707 & -.007 & .005 \\
\hline (Sum) Sugar & .090 & .538 & .004 & .008 \\
\hline (Sum) Starch & .018 & .890 & -.007 & .006 \\
\hline (Sum) Total fat & .164 & .306 & -.006 & .021 \\
\hline (Sum) Saturated & -.238 & .007 & -.047 & -.007 \\
\hline (Sum)Monounsaturated & .040 & .667 & -.019 & .030 \\
\hline (Sum)Polyunsaturated & -.016 & .798 & -029 & .022 \\
\hline (Sum)Fibreaoac & -.095 & $.020 * * *$ & -.033 & -.003 \\
\hline
\end{tabular}

$(*)$ for significant variables $(\mathrm{P}$ value $<0.05)$ 
All the significant variables were reassessed in one model with controlling for total physical activity. First, these variables were evaluated without total physical activity, then revaluated with physical activity. The result revealed that the physical activity is a potential confounder for the frequency of evening meals, favourite snacks frequency and sum of sugar from all foods including all beverages per day, as well as fibreaoac on their relationship to BMI. After adjusting for physical activity the correlation difference is greater than $10 \%$ for evening meal frequency. The difference is about $20 \%$ favorite snacks frequency. The adjustment difference in sum of sugar across all foods including all beverages per day and fibreaoac are more than $30 \%$. Thus, physical activity is a potential confounder for all these factors.

\section{Discussion}

The purpose of the present study was to determine the prevalence of being overweight and obese amongst 8-11 year old children in Cork city and county, and explore associations with self-reported oral hygiene practices and dietary choices. The current data demonstrated that more than $3 / 4$ of the study children are normal weight (75\%), while the remaining $1 / 4(25 \%)$ were overweight $(20 \%)$ or obese $(5 \%)$. The prevalence of the obesity in this study was consistent with another Irish study's findings (O'Neill et al., 2007), which was aimed to determine the prevalence of being overweight and obese in Irish children aged 8-11 years of age in 2007, using four different methods of weight to height measurement. The prevalence ranged from $4.1 \%$ to $16.3 \%$ (boys and girls, respectively). However, there was a slight different from North South survey, which showed the proportion of obesity $7 \%$ boys and $11 \%$ girls (Whelton et al., 2007).

Tooth brushing is a main method of oral hygiene care, using a toothbrush with fluoride toothpaste reduces oral diseases, such as dental caries and periodontal disease (Löe, 2000). The North South Survey of children in Ireland revealed that dental caries prevalence was high in fluoridated and non-fluoridated areas among children at age 5 and 15 years (Whelton et al., 2006). Dental caries prevalence in fluoridated areas was $36.9 \%$ and $73 \%$ (5 and 15 years respectively), while in non-fluoridated areas it was 54\% and $73.6 \%$ (5 and 15 years respectively) (Whelton et al., 2006).

Therefore, in this research, toothbrushing habits were assessed among BMI IOTF categories. The result showed that the majority of normal and overweight participants brush their teeth twice a day $(64.3 \%$ and $63.5 \%$, respectively). These findings are consistent with Oral 48 Hygiene among schoolchildren (HBSC) report 2006, which reported $62.5 \%$ of schoolchildren brush their teeth more than once a day (IRELAND, 2006). This trend has remained stable since 2002. Whereas, about $44 \%$ of the obese group brush their teeth twice a day and $15 \%$ do not usually brush their teeth. Furthermore, the study assessed the relationship between BMI and tooth brushing habits among the participants. The result presented a negative association between BMI and tooth brushing habits. This finding is inconsistent with another study, which showed a positive association. The study was conducted in Italy (Franchini et al., 2011). The study aimed to investigate the relationship between poor oral hygiene, gingivitis and obesity in young people, found that overweight and obese individuals have high gingival disease due to their bad attitude toward oral hygiene. Although the study recruited low sample size (90 subjects), it was well conducted and common factors were considered (metabolic and inflammatory factors) (Franchini et al., 2011).

However, the lack of an association between BMI and tooth brushing frequency was stated in a cross-sectional study (Prpic et al., 2012). Although they found a weak association between dental hygiene index and BMI, the correlation to tooth brushing frequency was not confirmed. This result was inconsistent with the current study. Study was well conducted (Prpic et al., 2012), sample size was lower than the present study. Furthermore, although this study observed worse dental health in high BMI individuals, the relationship of tooth brushing frequency could not be proven (Prpic et al., 2012). This result is contradicting, because tooth brushing is a significant factor in determining oral hygiene. Therefore, to observe the effect of tooth brushing frequency on BMI changes, a longitudinal study design needs to be conducted.

Diet is undoubtedly one of the most important determinants of BMI. Furthermore, it has a significant impact on oral health, particularly with regards to carbohydrate (sugar) (Burt and Pai, 2001; WHO Guidelines Approved by the Guidelines Review Committee, 2015). The current study evaluated the dietary choices among the participants. Diet was divided according to diet frequency, quality, and quantity. Then these categories were assessed in separate models and their relationship to BMI was investigated. In diet frequency, the breakfast, dinner, favorite drink and favorite snack frequencies were found to be significantly associated with increased BMI ( $\mathrm{p}$ value $<0.05)$.

The breakfast consumption was linked to reduced BMI, and the evidence for this relationship is growing(Utter et al., 2007). The relationship between breakfast consumption and BMI was significant and negatively associated with BMI in a New Zealand-based cross-sectional study (Utter et al., 2007). Suggesting that eating breakfast at home reduces the consumption of unhealthy snacks. However, this result was inconsistent with the current study's result. Although the current study predicted that eating breakfast at home reduces weight, the result was inverse, which found children who consumed breakfast had high BMI in parents' /guardians' response ( $<<0.005)$. However, when children were asked about frequency of breakfast consumption, the correlation was inverse: as breakfast decreased, the BMI increased, but this relationship was not statistically significant.

Snacks frequency was significantly associated to BMI. The same result was stated in a cross-sectional study in the UK. The study aimed to assess the association between BMI and snacks frequency. Several types of snack were assessed include crisps, sweets, chocolates and ice-creams and lower intakes of yoghurt and nuts (O'Connor et al., 2015). From the dental perspective, snacks, particularly sugary snacks, are correlated with dental caries (Sharma and Hegde, 2009). This is mostly related to eating habit, which leaves children having difficulty in maintaining their oral hygiene. The oral $\mathrm{pH}$ after eating sugar drops severely to critical levels within 40 minutes. If tooth brushing is not used, the acid produced by some bacteria starts to create the caries lesion (Henriksen and Kolset, 2007). For this reason, more attention should be given towards changing children's behaviours.

Parents /guardians' behaviour with respect to offering food as a reward for good behaviours. The results indicated that about $1 / 3(16.9 \%$ slight agree and $15.2 \%$ agree) of parents/guardians offered sweets for good behaviour. In addition, parents/guardians were asked if they supervised their children's eating of sweets, high fat, and junk foods (foods on top shelf of food pyramid) - almost $15 \%$ did not supervise their children's eating of sweets or high fat foods. While more than half of them (19.7\% slight agree and $47.5 \%$ agree) did not guide or regulate their children's eating of junk food. Junk food was significantly associated with BMI increase in this research.

Thus, the study highlights the importance of the parents' /guardians' role in controlling and guiding their children's eating, and they are able to choose a healthy diet for their 50 children if they are informed about health and unhealthy foods. Therefore, parents/guardian should be included in any educational and preventive programs. Role of parents in preventing childhood obesity was reviewed by Lindsay in (2006) (Lindsay et al., 2006). The researchers evaluated school-based obesity-prevention intervention programs that included parents as part of the intervention programs. Furthermore, the authors reviewed the programs that aimed to improve the parents' ability to shape their children's behavior. The researchers concluded that childhood obesity prevention programs needs multifaceted and wide community-based intervention, and parents should have a critical role in this. In addition, the prevention programs needs to be directed towards parents at an early age to develop healthful practice in and outside the home(Lindsay et al., 2006). 
Furthermore, parents/guardians should be informed about the importance of personal factors include oral health education, attitude, literacy, knowledge and behavior (such as oral hygiene and snacking). Therefore, parents/guardians should be included in any oral or general health promotion to ensure its effectiveness.

Diet quantity was assessed in this research and its relationship to BMI was investigated. The result revealed that the total sugar consumption from all foods including all beverages per day and the total Kcal of all foods (irrelevant remove) were significantly correlated to BMI. They can be indicators for BMI increase. The result of the present study is consistent with the findings of other studies (Martins et al., 2014; Millar et al., 2014). Both studies aimed to assess the relationship between BMI and Sugar intake. Finding that sugar intake was strongly associated with BMI Millar et al., (2014) conducted a longitudinal study to observe the relationship between BMI and sugar consumption frequency over time. They found that with each increase of sugar consumption, BMI increased $(0.015$ unit, $p<0.001)$. Hence, the association in Millar et al. was positive, which is consistent with th7is study's findings that showed a positive association between BMI and sugar intake $35 \%$ (coefficient correlation $=0.353$ ).

In the present study the strength of the association of evening meals, favorite snacks, favorite drinks frequency and the sum of sugar from all foods including all beverages per day and fibreaoac with BMI were changed after controlling for physical activity. The strength of the association was increased for favorite snacks, favorite drinks and total sugar from all foods, while it was decreased for dinner meals and fibreaoac after adjustment. Thus, the present study highlights the importance of the physical activity in assessment of the relationship between BMI and dietary choices.

\section{Weaknesses and limitations}

This study has some weaknesses, such as the cross-sectional design, which cannot establish the temporal sequence and observe BMI changes over time. Therefore, a longitudinal study design should be conducted, in order to establish temporal relationship, dose-response relationship, and observe changes over the time. However, the study sample is large, which increases study power. In addition, the study findings are consistent with the findings of several previous studies.

\section{Conclusion}

The study concluded that breakfast frequency, dinner meals, snacks frequency, total sugar from all foods including all beverages per day, fibreaoac and sum Kcal of all foods are indicators for weight increase. Therefore, the study concludes that tooth brushing frequency has a significant part to play not only in maintaining oral health, but also should be considered with respect to BMI changes. Furthermore, dietary choices, especially sweets have an important role in being overweight and suffering obesity.

Furthermore, sweets have a significant role to play in the creation of caries lesion. Moreover, this study suggested that parents/guardians could play a significant role in the prevention of both conditions (obesity and dental caries). This can be most successful if they are targeted in an educational and prevention program.

\section{Recommendations}

It is recommended that any future preventive programs should aim at raising both the general health awareness and improving oral health. In addition, strategies should be included, on a multidisciplinary level, and should integrate parents to prevent and reduce both obesity and dental caries at an early age. In addition, raise parents awareness towards the common risk factors include diet (sugars) and lifestyle (physical activity). Additionally, it is recommended that a longitudinal study should be conducted in the future to establish the changes in the BMI and to provide good evidence.

\section{References}

[1] Burt BA, Pai S (2001). Sugar consumption and caries risk: a systematic review. Journal of dental education 65(10):1017-1023. https://doi.org/10.1002/j.0022-0337.2001.65.10.tb03444.x.

[2] Council NR (1989). Diet and health: implications for reducing chronic disease risk: National Academies Press.

[3] Franchini R, Petri A, Migliario M, Rimondini L (2011). Poor oral hygiene and gingivitis are associated with obesity and overweight status in paediatric subjects. Journal of clinical periodontology 38(11):1021-1028. https://doi.org/10.1111/j.1600-051X.2011.01770.x.

[4] Gupta P, Gupta N, Pawar AP, Birajdar SS, Natt AS, Singh HP (2013). Role of sugar and sugar substitutes in dental caries: a review. ISRN dentistry 2013(519421. https://doi.org/10.1155/2013/519421.

[5] Henriksen HB, Kolset SO (2007). [Sugar intake and public health]. Tidsskrift for den Norske laegeforening : tidsskrift for praktisk medicin, ny raekke 127(17):2259-2262.

[6] IRELAND H (2006). Oral Hygiene among schoolchildren in Ireland HBSC IRELAND.

[7] Jeor STS, Howard BV, Prewitt TE, Bovee V, Bazzarre T, Eckel RH et al. (2001). Dietary protein and weight reduction. Circulation 104(15):18691874. https://doi.org/10.1161/hc4001.096152.

[8] Lindsay AC, Sussner KM, Kim J, Gortmaker S (2006). The role of parents in preventing childhood obesity. The Future of children:169-186. https://doi.org/10.1353/foc.2006.0006.

[9] Löe H (2000). Oral hygiene in the prevention of caries and periodontal disease. International dental journal 50(3):129-139. https://doi.org/10.1111/j.1875-595X.2000.tb00553.x.

[10] Martins RJ, Moimaz SAS, Silva MR, Saliba O, Garbin CAS (2014). Body mass index, dental caries and sugar intake in 2-5 year-old preschoolers. Brazilian Journal of Oral Sciences 13(3):209-212. https://doi.org/10.1590/1677-3225v13n3a09.

[11] Millar L, Rowland B, Nichols M, Swinburn B, Bennett C, Skouteris H et al. (2014). Relationship between raised BMI and sugar sweetened beverage and high fat food consumption among children. Obesity (Silver Spring, Md) 22(5):E96-103. https://doi.org/10.1002/oby.20665.

[12] O'Connor L, Brage S, Griffin SJ, Wareham NJ, Forouhi NG (2015). The cross-sectional association between snacking behaviour and measures of adiposity: the Fenland Study, UK. Br J Nutr 114(8):1286-1293. https://doi.org/10.1017/S000711451500269X.

[13] O'Neill J, McCarthy S, Burke S, Hannon E, Kiely M, Flynn A et al. (2007). Prevalence of overweight and obesity in Irish school children, using four different definitions. European Journal of Clinical Nutrition 61(6):743-751. https://doi.org/10.1038/sj.ejcn.1602580.

[14] Pitts N, Amaechi B, Niederman R, Acevedo AM, Vianna R, Ganss C et al. (2011). Global oral health inequalities: dental caries task group--research agenda. Advances in dental research 23(2):211-220. https://doi.org/10.1177/0022034511402016.

[15] Prpic J, Kuis D, Pezelj-Ribaric S (2012). Obesity and Oral Health - Is There an Association? Coll Anthropol 36(3):755-759. 
[16] Reilly D, Boyle C, Craig D (2009). Obesity and dentistry: a growing problem. British dental journal 207(4):171-175. https://doi.org/10.1038/sj.bdj.2009.717.

[17] Selwitz RH, Ismail AI, Pitts NB (2007). Dental caries. Lancet (London, England) 369(9555):51-59. https://doi.org/10.1016/S0140-6736(07)600312 .

[18] Sharma A, Hegde A (2009). Relationship between body mass index, caries experience and dietary preferences in children. Journal of Clinical Pediatric Dentistry 34(1):49-52. https://doi.org/10.17796/jcpd.34.1.17364206hqm0477h.

[19] Utter J, Scragg R, Mhurchu CN, Schaaf D (2007). At-home breakfast consumption among New Zealand children: associations with body mass index and related nutrition behaviors. Journal of the American Dietetic Association 107(4):570-576. https://doi.org/10.1016/i.jada.2007.01.010.

[20] Whelton H, O'Mullane D, Harding M, Guiney H, Cronin M, Flannery E et al. (2006). North South survey of children's oral health in Ireland 2002. 11.

[21] Whelton H, Harrington J, Crowley E, Kelleher V, Cronin M, Perry IJ (2007). Prevalence of overweight and obesity on the island of Ireland: results from the North South Survey of Children's Height, Weight and Body Mass Index, 2002. BMC public health 7(1):1. https://doi.org/10.1186/14712458-7-187.

[22] WHO Guidelines Approved by the Guidelines Review Committee (2015). In: Guideline: Sugars Intake for Adults and Children. Geneva: World Health Organization Copyright (c) World Health Organization, 2015. 